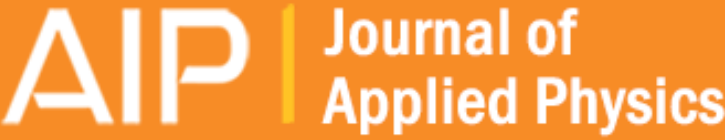

\section{A systematic study of the effect of casting solvent on the bulk properties of linear SBS block copolymers}

Garth L. Wilkes, Shiriam Bagrodia, Zohar Ophir, and John A. Emerson

Citation: Journal of Applied Physics 49, 5060 (1978); doi: 10.1063/1.324449

View online: http://dx.doi.org/10.1063/1.324449

View Table of Contents: http://scitation.aip.org/content/aip/journal/jap/49/10?ver=pdfcov

Published by the AIP Publishing

\section{Articles you may be interested in}

Detailed molecular dynamics studies of block copolymer directed self-assembly: Effect of guiding layer properties

J. Vac. Sci. Technol. B 31, 06F302 (2013); 10.1116/1.4821652

A study for the static properties of symmetric linear multiblock copolymers under poor solvent conditions

J. Chem. Phys. 136, 094902 (2012); 10.1063/1.3689303

Effect of chain topology of block copolymer on micellization: Ring versus linear block copolymer

J. Chem. Phys. 118, 8468 (2003); 10.1063/1.1566436

Notes: Extension of the Contour Plotting Method for Representing Linear Viscoelastic Loss Properties of SBS

Triblock Copolymers

Trans. Soc. Rheol. 21, 157 (1977); 10.1122/1.549459

Application of NMR to the study of molecular motion in SBS copolymers J. Appl. Phys. 45, 3441 (1974); 10.1063/1.1663798

MIT LINCOLN LABORATORY CAREERS

Discover the satisfaction of innovation and service to the nation
- Space Control

- Air \& Missile Defense

- Communications Systems \& Cyber Security

- Intelligence, Surveillance and Reconnaissance Systems

- Advanced
Electronics
- Tactical Systems
- Homeland
Protection
- Air Traffic Control

- Air Traffic Control

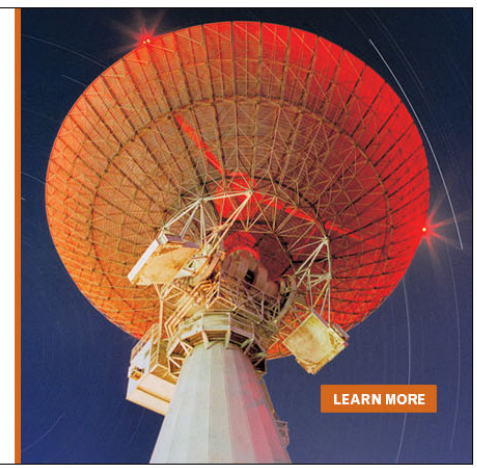




\title{
A systematic study of the effect of casting solvent on the bulk properties of linear SBS block copolymers
}

\author{
Garth L. Wilkes ${ }^{\text {a) }}$ \\ Department of Chemical Engineering, VPI\&SU, Blacksburg, Virginia 24061 \\ Shiriam Bagrodia and Zohar Ophir \\ Polymer Materials Program. Department of Chemical Engineering, Princeton University, Princeton, \\ New Jersey 08540 \\ John A. Emerson \\ Western Electric Research Center, Princeton, New Jersey 08540 \\ Using a well-characterized series of styrene-butadiene triblock copolymers, a systematic study was carried \\ out to determine the influence of casting solvent, used for film preparation, on the final mechanical \\ properties. The results clearly indicate that in the range of styrene contents of $30-40 \mathrm{~mole} \%$, the \\ modulus behavior varies greatly as the solubility parameter $\delta$ of the casting solvent is varied. Specifically, \\ the modulus increases as $\delta$ increases. At lower or higher styrene content, the properties are influenced \\ but to a much lesser degree. Interpretation of the measured mechanical behavior is given in light of \\ morphological data obtained by rheo-optical methods.
}

PACS numbers: $61.40 . \mathrm{Km}, 81.30 \mathrm{Mh}, 81.40 . \mathrm{Jj}, 64.75 .+\mathrm{g}$

\section{INTRODUCTION}

To date there have been numerous studies on the structure-property relationships of ABA triblock copolymers, with particular emphasis on those of the SBS type. ${ }^{j-9}$ Past studies have focused, for example, on such topics as relative $A / B$ ratio and block lengths. It has also been realized that the nature of the preparative conditions used for obtaining films can influence the final morphology, and therefore the final properties. ${ }^{1-4,7,10-13}$ Regarding preparative methods, the two basic procedures are to produce films by thermal moulding or by solution casting. It is the latter method that concerns this paper. In this paper we will address this problem by noting how the morphology and the mechanical and rheooptical behavior of a systematic series of linear SBS systems changes with casting solvent. Hopefully, our results will aid those using solvent casting as a preparative means in future investigations in that the final properties may be greatly influenced by choice of solvents. The concepts discussed should also be applicable to other triblock and multiblock systems.

TABLE I. Molecular characterization data on the styrene-butadiene block copolymers used in the study.

\begin{tabular}{llrl}
\hline \hline $\begin{array}{l}\text { Mole ratio } \\
\text { butadiene/styrene }\end{array}$ & $\bar{M}_{w}{ }^{\mathrm{a}}$ & \multicolumn{1}{c}{$\overline{\boldsymbol{M}}_{n}^{\mathrm{a}}$} & $\begin{array}{l}\text { Inherent } \\
\text { viscosity }\end{array}$ \\
\hline $90 / 10$ & 110000 & 95000 & 1.32 \\
$80 / 20$ & 117000 & 100000 & 1.05 \\
$70 / 30$ & 111000 & 96000 & 0.88 \\
$60 / 40$ & 100000 & 88000 & 0.80 \\
$50 / 50$ & 98000 & $\mathbf{8 7 0 0 0}$ & 0.79 \\
$25 / 75$ & 96000 & $\mathbf{8 3 0 0 0}$ & 0.56 \\
\hline \hline
\end{tabular}

a Determined from calibrated GPC curves.

'In THF at $25^{\circ} \mathrm{C}$.

a 'To whom correspondence should be sent.

\section{EXPERIMENTAL Materials and film preparation}

The styrene-butadiene ( SBS ) triblock copolymers under study were obtained through the courtesy of H. L. Hsieh of Phillips Petroleum Co. The styrene content ( mole\%) varied from 10 to $75 \%$ in a systematic manner. The average number molecular weight of each SBS copolymer was around $90000 \mathrm{~g} / \mathrm{mole}$. The solubility parameters of the styrene block and butadiene blocks were assumed to be 9.4 and 8.6 , respectively. These values are certainly reasonable in light of the present literature. ${ }^{2.14}$ Accordingly, a number of casting solvents covering the entire range of the solubility parameter $(\delta)$ from 7.8 to 10.0 were selected in this study; the previous work of Samuels and Wilkes assisted in the selection of these solvents 2 . Tables I and II give the details with respect to the polymer samples and solvents used. Thin copolymer films were solution cast from each solvent on a Teflon surface at room temperature. The films were then placed under vacuum for a few days to minimize traces of solvents.

\section{Properties evaluation}

A standard table Model TMS 84 Instron was used to determine the mechanical properties for all the samples. All

TABLE II. Solvents ( and solubility parameters ) used for casting copolymer films.

\begin{tabular}{lc}
\hline \hline Solvent & $\begin{array}{l}\text { Solubility } \\
\text { parameter }(\delta)\end{array}$ \\
\hline Methyl cyclohexane (MCH) & 7.8 \\
Cyclohexane ( CH ) & 8.2 \\
Carbon tetrachloride & 8.6 \\
Toluene & 8.9 \\
Chloroform & 9.3 \\
1,1,2,2 tetrachloroethane ( TCE) & 9.7 \\
1,4 dioxane & 10.0 \\
\hline
\end{tabular}


measurements were made at room temperature. Samples were $3.5-4.0 \mathrm{~cm}$ in the length and $0.5 \mathrm{~cm}$ in width. A crosshead speed of $10.0 \mathrm{~cm} / \mathrm{min}$ was used.

A rheovibron DVDII ( Toyo Instruments ) was utilized for obtaining the dynamic mechanical spectra at a frequency of $110 \mathrm{~Hz}$.

Birefringence data were obtained in conjunction with stress-relaxation measurements for purposes of determining the stress-optical ( SOC) and strain-optical ( StOC) coefficients. The birefringence data were measured by a Babinet compensator.

Small-angle X-ray scattering ( SAXS) data were obtained at ambient temperature using a PDP-8A controlled Kratky camera. For these studies, the entrance slit width was $30 \mu \mathrm{m}$, while the receiving slit width was $75 \mu \mathrm{m}$ with a length of $10 \mathrm{~mm}$. Background scans were made after each sample run.

Transmission electron micrographs were obtained from osmium tetraoxide stained ultrathin films that had been microtomed at liquid-nitrogen temperature. Two types of sections were investigated, those microtomed perpendicular to the cast film plane and sections cut parallel to the cast film plane.

\section{RESULTS AND DISCUSSION}

For a given casting solvent, the effect of styrene content on mechanical properties is as expected. Figure 1( a ) shows, for example, the initial stress-strain curves of films of different styrene content all having been cast from carbon tetrachloride $(\delta=8.6)$. As anticipated, as the styrene content increases the modulus increases as clearly noted from the increase of the initial slope of the stress strain curve. Furthermore, above $20 \%$ styrene, the curves display a distinct yield point and in fact display a necking behavior as well. At $75 \%$ styrene, the material for this casting solvent was rather brit- tle and did not display significant extensibility. The cause of this systematic stiffening of the material with styrene content is no surprise and arises due to the development of a continuous glassy styrene phase as the mole percent of the styrene component increases. At 75 mole\% styrene, the material behavior is indeed rather similar to styrene homopolymer when measured under similar conditions in that it is high modulus and displays low strain to break.

The more interesting data is to note how the mechanical behavior changes for a material of given styrene content when the casting solvent is changed. A very distinct example of this is shown in Fig. 1(b) which illustrates this change in behavior for the 30 mole\% styrene material. A number of interesting points are noted from these data gathered at ambient temperature. First, as $\delta$ increases, the modulus increases significantly ( quantitative values presented shortly). Also, the yield point behavior is also induced for the higher $\delta$ values. In general, the strain hardening process seems to go through a maximum near $\delta$ of 8.6 although more tests would be necessary to confirm this observation. The basic trends observed here were reported earlier by Wilkes and Stein on the well known Kraton ${ }^{\mathrm{R}}$ SBS block copolymer material ${ }^{4}$ and also by Beamish et al. ${ }^{10}$ and Wilkes et al. ${ }^{1}$ on experimental systems in more recent publications. Before presenting any molecular explanation of these data, we present Figs. 2( a ) and 2(b) which show Young's modulus versus styrene mole $\%$ and $\delta$, respectively. In each plot, however, the effect of both of these latter two variables can be noted. It is interesting to observe that in Fig. 2(a) there is little effect of changing $\delta$ on the lowest mole\% styrene material yet the effects are considerable when above $20 \mathrm{~mole} \%$. In Fig. 2(b), the more significant result is that the effect of changing $\delta$ for a given styrene content is most pronounced for the $30 \%$ styrene sample-in fact the modulus increases by a factor of 20 from values of $\sim 20 \mathrm{MPa}(\delta=7.8)$ to a value of $\sim 220 \mathrm{MPa}(\delta=10.2)$. As discussed later, this major
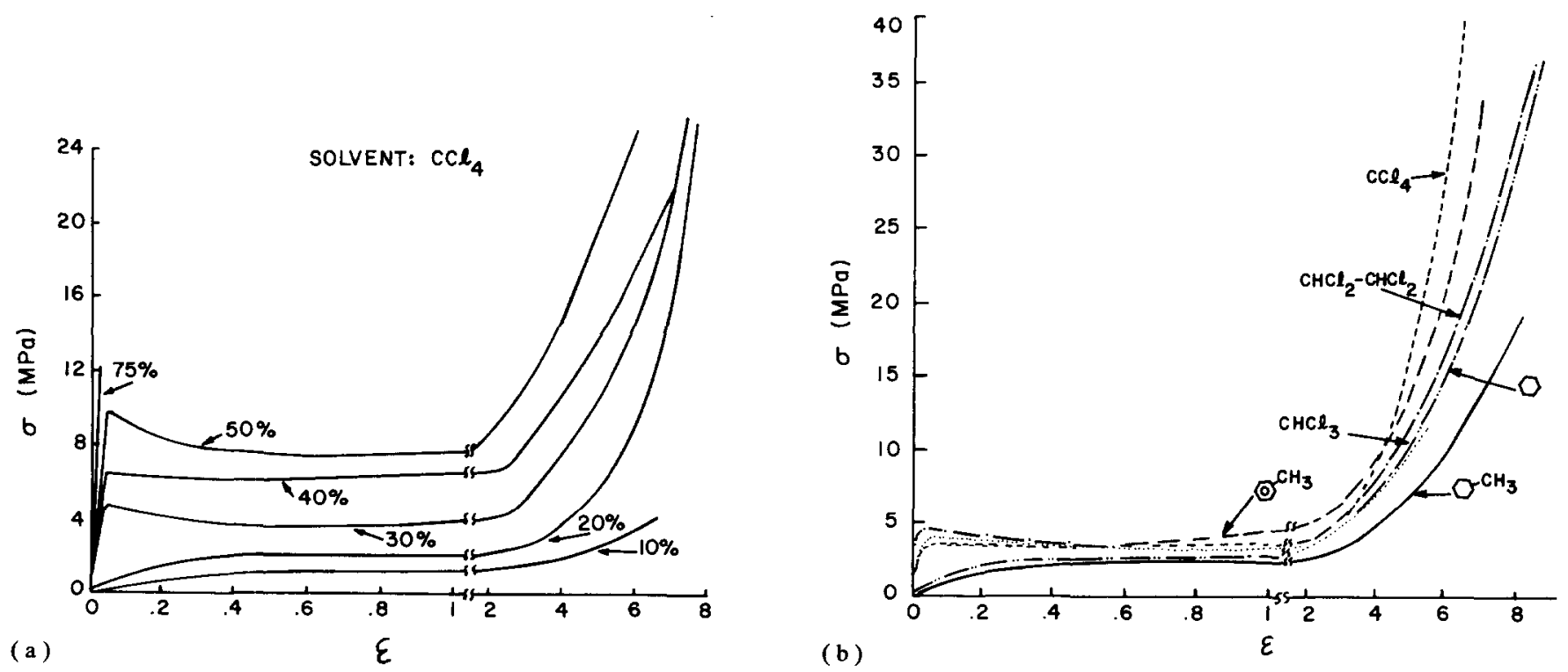

FIG. 1. (a ) Stress-strain plotted for block copolymers of different styrene contents. The casting solvent was carbon tetrachloride ( $\left.\mathrm{CCl}_{4}\right)$ in all cases. (b) Stress-strain plots for a block copolymer having a 30 mole\% styrene content cast from different solvents. 

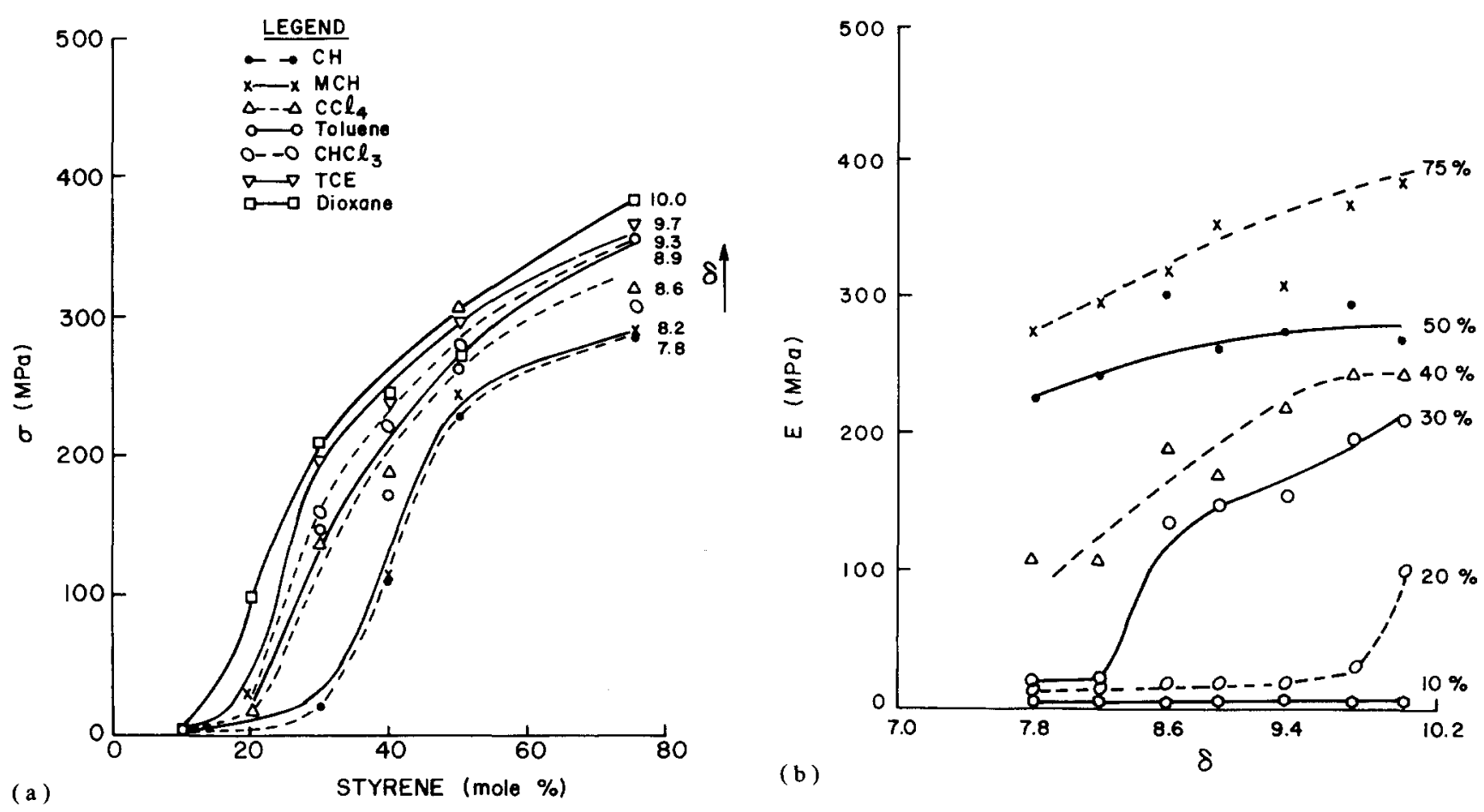

FIG. 2. (a ) Young's modulus plotted as a function of styrene mole\%. Each curve represents data obtained from a given solvent. (b) Young's modulus plotted against $\delta$ for different styrene content copolymers.

change occurs in this region due to the fact that this composition ratio is apparently particularly sensitive to changes in domain morphology ( spherical, cylindrical, or lamellar ) with $\delta$ than are the other ratios. However, as will be indicated, the effects cannot all be accounted for when only changes in morphology are considered. In light of the data given in Fig. 2( b ), it is appropriate to mention that due to the strong dependence of mechanical properties on casting solvent, using only the styrene content as a predictor of behavior is clearly an oversimplification. Such approaches have been made $^{15}$ but the results should be viewed with skepticism.

With respect to Fig. 2( b ), it may be noted that the data also suggest some resemblance to that obtained from temperature-frequency ( time) studies of mechanical properties. In this case raising mole\% styrene is analogous to lowering temperature, whereas raising $\delta$ is analogous to an increase in frequency. Indeed, one may intuitively suspect that this analogy has some merit, yet at this time we have not attempted the usual shift procedures due to the lack of a theoretical basis for this approach.

To display the yield behavior in more detail, Fig. 3 shows the yield stress, $\sigma_{y}$, versus mole $\%$ styrene. From these data one sees that the yield stress is not particularly sensitive to $\delta$. However, it seems to display a maximum in behavior with an increase in the mole\% styrene at a given $\delta$. Figure 4 shows the breaking stress, $\sigma_{b}$, as a function of percent styrene for the materials cast at a given $\delta$ value. While there is some hesitancy to accept these ultimate strength data ( due to few samples tested ), the data imply that there is a maximum in $\sigma_{b}$ with styrene content and yet it is difficult to make a correlation between these two parameters. As shown in Fig. 5, a trend exists for the degree of permanent set with styrene content immediately following an extension to break. There again is a strong correlation with $\delta$ and it appears that a lower $\delta$ will give a lower degree of set than do the higher $\delta$ solvents.

In investigating the mechanical behavior at other temperatures, dynamic mechanical techniques were utilized. The storage modulus and $\tan \delta$ data are given in Fis. 6(a) and 6 (b), respectively, for the 30 mole\% styrene samples. ( This material was used since it displayed the largest change in modulus with change in $\delta$.) As expected, the mechanical spectra does indeed indicate the major effects of casting solvent. This being that, in general, the material displays a higher value of $E^{\prime}$ above the $T_{g}$ of the butadiene block for samples cast from solvents with higher $\delta$. Furthermore, as the value

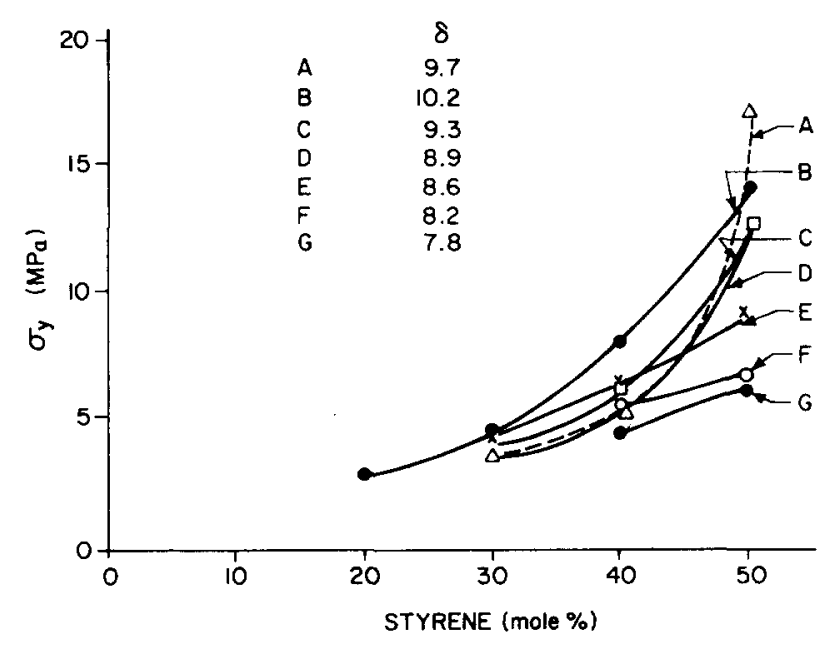

FIG. 3. Yield stress plotted against mole\% styrene content. Each line represents a different casting solvent, i.e., $\delta$. 


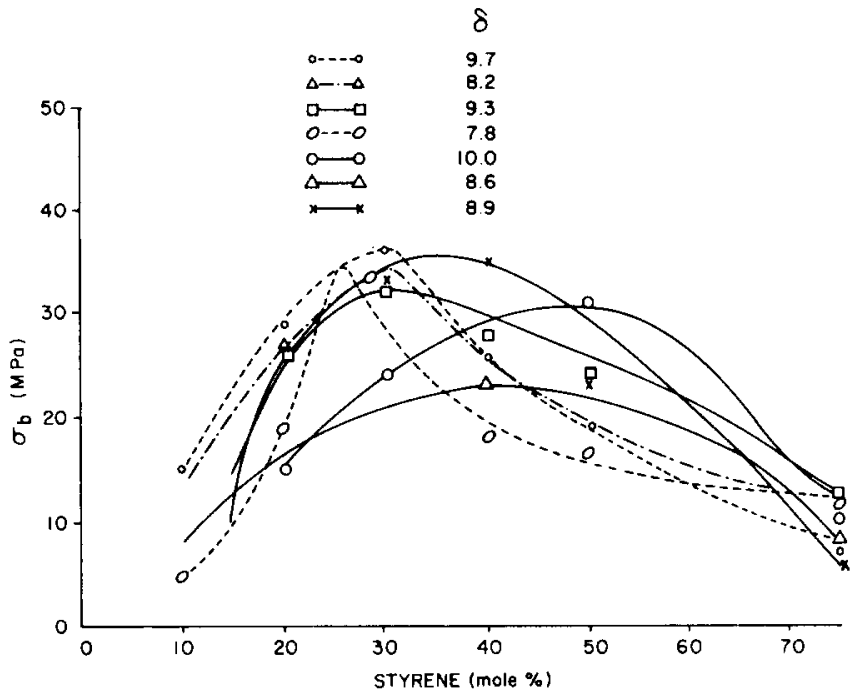

FIG. 4. Breaking stress plotted against $\delta$ for different styrene content copolymers.

of $\delta$ increases, the magnitude of the butadiene $\tan \delta$ peak tends to narrow and diminish in amplitude, whereas the reverse appears for the higher styrene glass transition. No strong indication of any intermediate peak was noted in this study (or those in the past from this laboratory) as some others have claimed to detect. ${ }^{16}$

While the nature of the morphological texture of the domain structure is clearly important with respect to mechanical properties, the degree and perfection of phase separation of the two components are two other important variables affecting mechanical behavior. The dynamic mechanical data suggest, but do not prove, that differences in these parameters exist with different casting solvent. To attempt to shed some light on this question, the use of stress- and strain-optical measurements as well as TEM and SAXS studies were made.

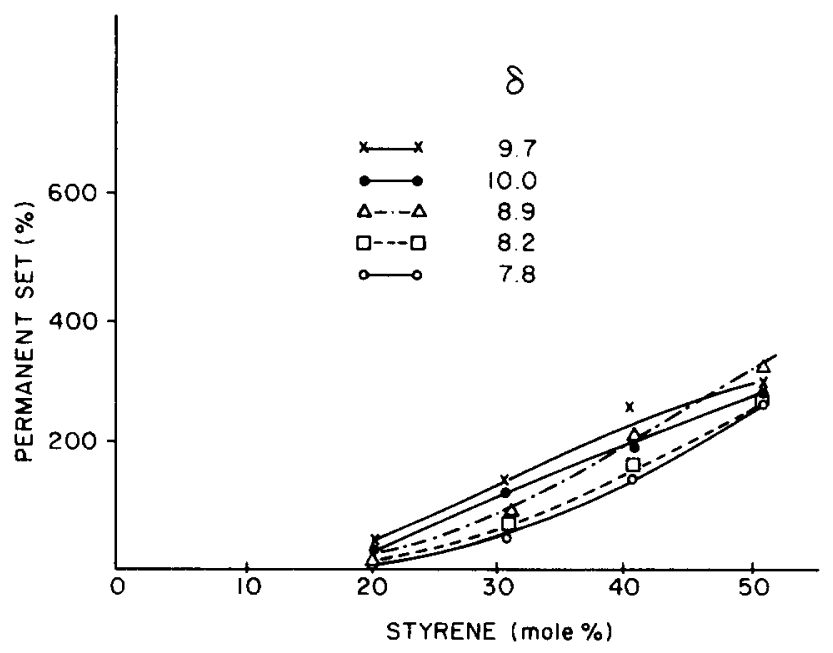

FIG. 5. Permanent set ( $\%$ ), as determined immediately after break, plotted against mole\% styrene. Each line represents a single casting solvent, i.e., fixed $\delta$.

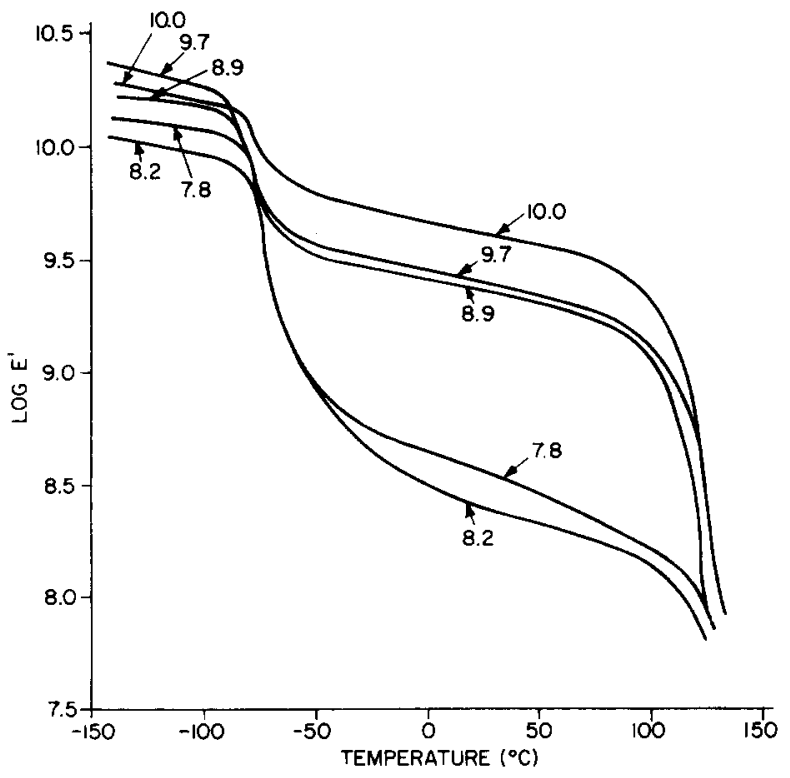

( a )

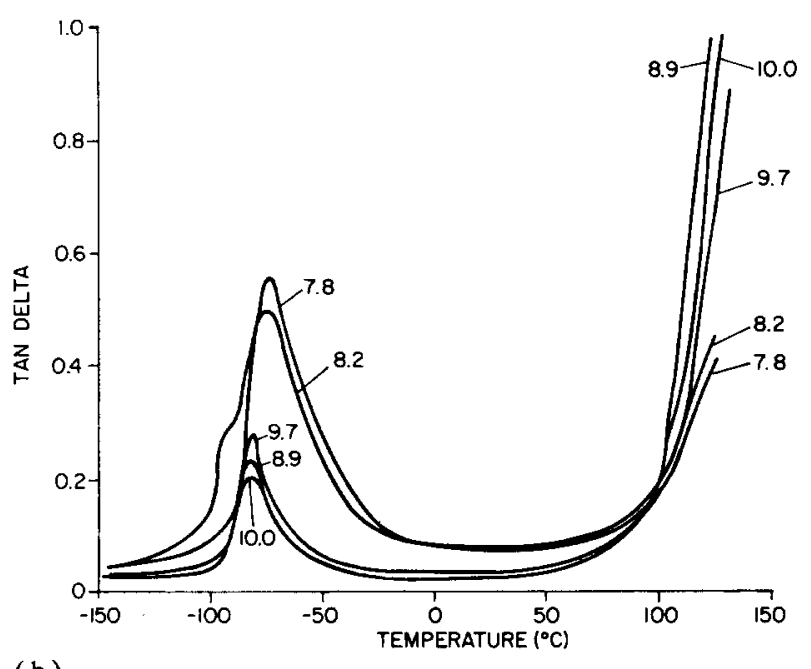

(b)

FIG. 6. (a) Plot of the real part of Young's dynamic modulus, $E^{\prime}$, against temperature for the 30 mole\% styrene copolymer cast from solvents of different $\delta .(110 \mathrm{~Hz}$ ). (b) Plot of $\tan \delta$ against temperature for the $30 \mathrm{~mole} \%$ styrene copolymer cast from solvents of different $\delta .(110 \mathrm{~Hz})$.

In carrying out the TEM investigations, again only the 30 mole\% styrene sample was investigated. Also, only those films prepared from methyl cyclohexane $(\delta=7.8)$, toluene $(\delta=8.9)$, and 1,4 dioxane $(\delta=10.0)$ were utilized. As stated earlier, two types of ultrathin sections were prepared, one normal to the film plane and another parallel to the film plane. The double sectioning was felt necessary in light of the inability to confirm a given morphology if observed in only a single plane.

Figures 7(a )-7( c) show typical examples of the sections prepared normal to the film plane, while Figs. 8( a )8 (c) show examples prepared parallel to the plane of the cast film. Two important observations can be qualitatively made: ( 1 ) The morphology in all cases is basically cylindrical and/or lamellar but surprisingly no indication of any spherical domain structure is present as was somewhat ex- 


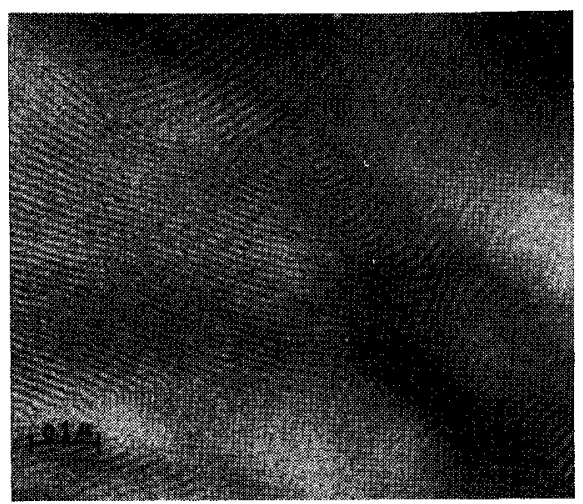

(a)

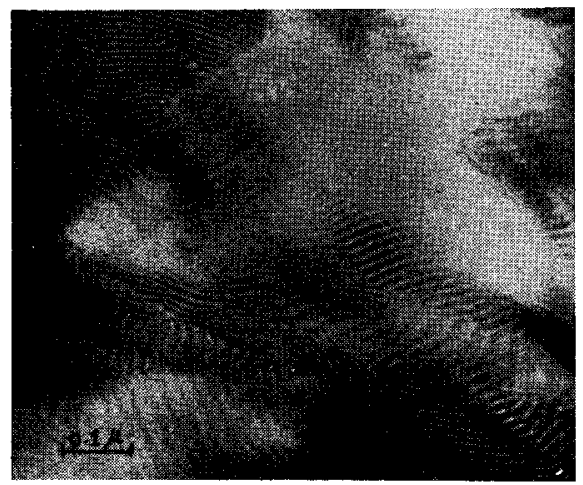

(b)

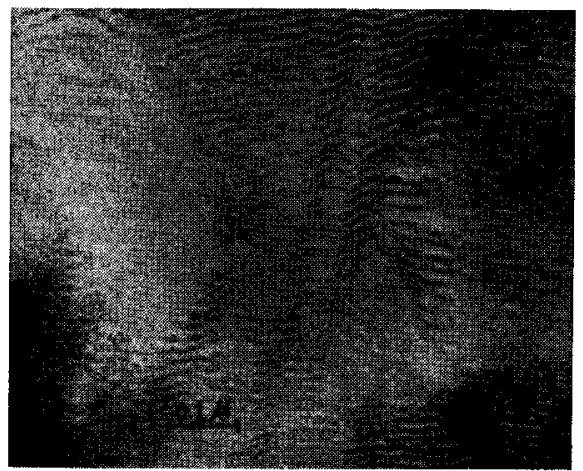

(c)

FIG. 7. Transmission electron micrographs of the 30 mole $\%$ styrene material cast from ( a ) cyclohexane, (b) toluene, and (c) dioxane. Micrographs are from sections all microtomed perpendicular to the plane of the cast film.

pected for at least the low $\delta$ films. (2) Indeed while the morphological texture does show some differences with $\delta$ values, particularly for the parallel sections, the only apparent distinction is that the higher $\delta$ film seems to show less distinct phase separation than the other films-for example, compare Fig. 7(c) with Figs. 7( a ) and 7(b). This qualitative observation, while assisting in possibly understanding the changes in mechanical properties with $\delta$, does not conclusively confirm differences in the phase separation behavior since it is well understood that TEM observations are confined to a small area and what is observed may be dependent on preparative methods. For this reason we obtained SAXS scans on these same materials. The results are shown in Figs. 9 where it is clear each film displays a maximum which reflects the phase separation behavior. The point of particular importance is that the peak shifts somewhat with $\delta$ and clearly the peak sharpness decreases as $\delta$ increases. The latter effect distinctly suggests that the degree of phase separation is less in films of higher $\delta$ as indeed the TEM micrographs suggests. We have not pursued the in-depth treatment of the SAXS data on these block systems; howev$\mathrm{er}$, it is likely that an invariant analysis such as that used by Hashimoto et al. ${ }^{17}$ might well give further quantitative support to the above conclusion. At this point then it is indicated that much of the changes in mechanical properties with $\delta$ are more an effect of changes in phase separation rather than a major change in the morphological texture of the domain structure-at least for the 30 mole\% styrene composition.

As a final approach to understanding the mechanical properties of these materials and how they are affected by $\delta$, stress-optical coefficient ( SOC) and strain-optical coefficient ( StOC ) measurements were made on many of the ma-

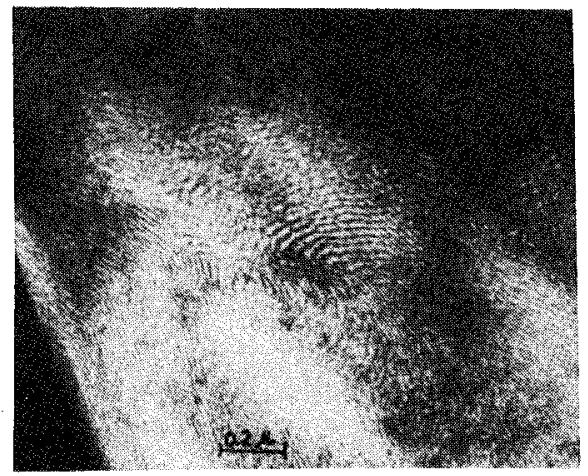

(a)

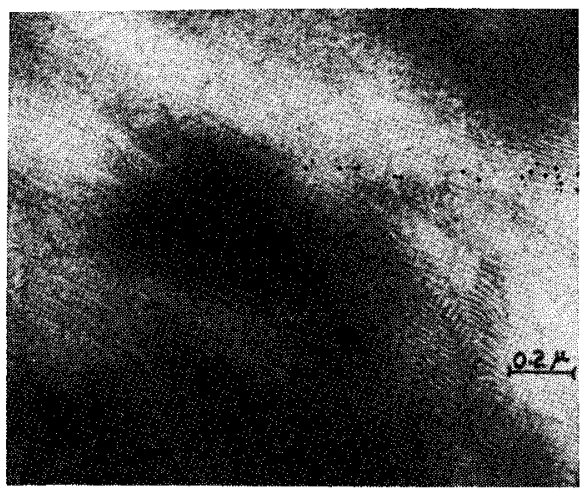

(b)

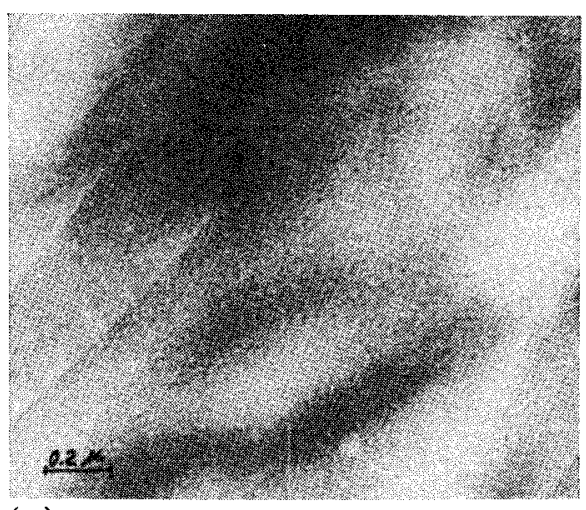

(c)

FIG. 8. Transmission electron micrographs of the 30 mole\% styrene material cast from (a) cyclohexane, (b) toluene, and (c) dioxane. Micrographs are from sections microtomed parallel to the plane of the cast film. 


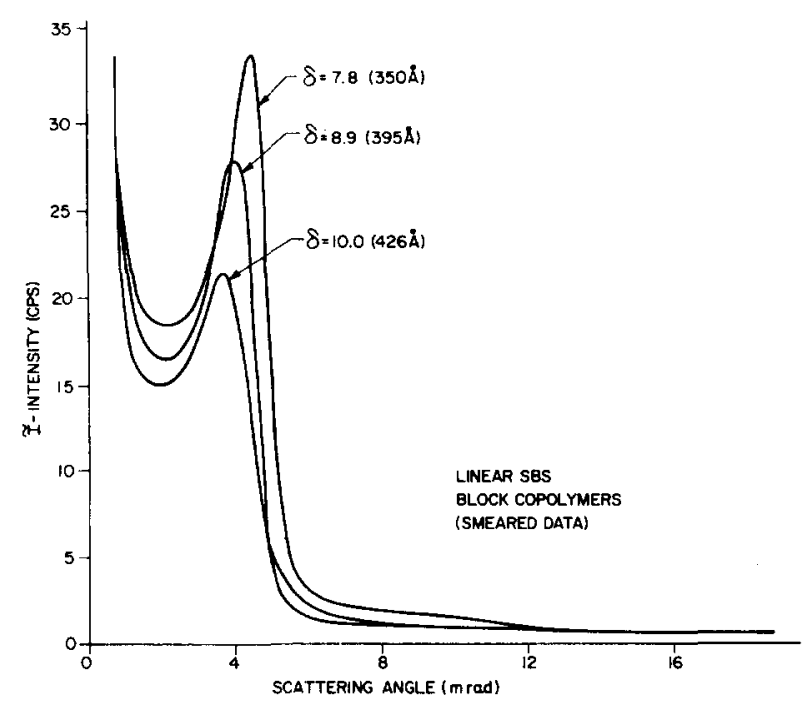

FIG. 9. Plot of the small-angle $x$-ray scattering intensity, $\widetilde{I}$, versus scattering angle for the 30 mole\% styrene copolymer cast from three different solvents. The $\delta$ values of the respective solvents are given as is the Bragg spacing for each scattering peak as obtained from the smeared data.

terials at selected extension levels. The initial extension curve was used since this reflects the initial structure. As is well known, films cast from higher $\delta$ sovlents show considerable softening on the second or higher extensions. ${ }^{7,9}$

Because of nonuniform deformation ( necking) of the higher styrene content materials, as well as those cast from high $\delta$ volents, bench marks were placed on the samples to ensure that the SOC and StOC parameters were determined at equivalent strain levels. Two levels were selected-30\% and $200 \%$ elongation. The data presented here will be for the $200 \%$ elongation; however, the basic behavior to be presented is similar for the $30 \%$ extension data. Figures 10 (a) shows the plot of SOC versus $\delta$ for the $30 \%$ styrene content sample, while Fig. 10(b) shows the value of SOC as a function of styrene content. In Figs. 11( a ) and 11( b ) the StOC is plotted for the same variables respectively. Looking first at the SOC and StOC data as a function of styrene content it is noted that each optical parameter decreases as a function of styrene. The basic fact that both decrease is expected in light of the SOC behavior of each of the block components. That is, the SOC values of rubbery butadiene ( of the chain microstructure in these systems ) glassy styrene and rubbery styrene are such that as the styrene content increases, the overall value of the $\mathrm{SOC}$ will decrease if both components are active mechanically, that is, they are load bearing. In fact, as Wilkes and Samuels have shown earlier, if a series model is used for describing the rheo-optical behavior, the SOC of these system should decrease linearly with styrene content ${ }^{5}$. Such an oversimplified model clearly has its limitations, but if even both components do not bear the applied stress equally, the SOC would still be expected to decrease with styrene content.

Similarly, arguments for the decrease in StOC with styrene content can be made but again the predicted results will depend on the nature of the model selected, parallel or series, and upon the distribution of strain for each component. For example, assuming a distinct two-phase model where the phases acted in parallel so that the strain, $\epsilon$ on both components are equal, we can write that

$$
\mathrm{StOC}_{\mathrm{system}}=\frac{\Sigma \Delta_{i} \phi_{i}}{\epsilon}=\frac{\Delta_{S} \phi_{S}}{\epsilon}+\frac{\Delta_{B} \phi_{B}}{\epsilon},
$$

where $\Delta_{S}$ and $\Delta_{B}$ are the birefringence values of the styrene and butadiene components, respectively, and $\phi_{S}$ and $\phi_{B}$ are the respective volume fractions. In light of the fact that for a given strain level, $\Delta_{B}$ would exceed $\Delta_{S}$, it is clear that the $\mathrm{StOC}_{\text {system }}$ would decrease in a linear fashion with styrene content. Again, such a model is clearly too simplified for two main reasons. The first and most significant is that a simple parallel addition of elements is not likely to be truely descriptive of the system in light of expected changes in morphology with styrene content. Specifically, for lower styrene contents, the butadiene component is expected to be the continuous matrix so that the majority of the strain will be distributed to this phase as compared to the spherical styrene

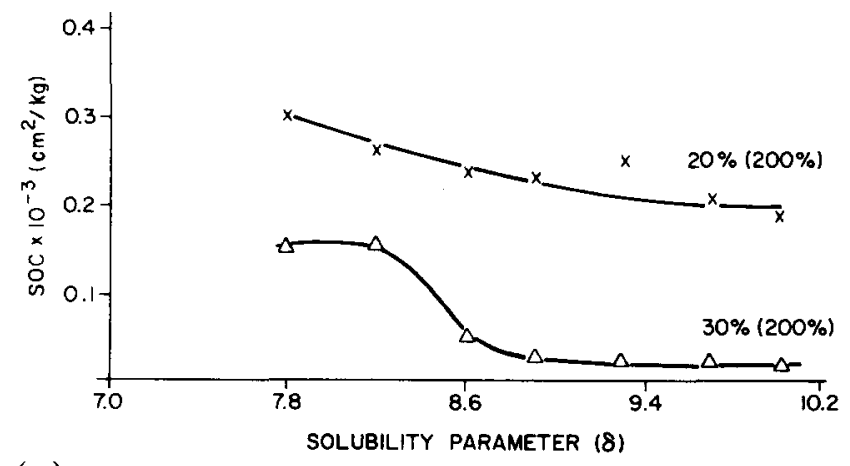

(a)

(10 FIG. 10. (a) Plot of the SOC for the 20 and 30 mole\% styrene copolymers versus $\delta$ as determined for $200 \%$
styrene. Films were all cast from carbon tetrachloride, $\delta=8.6$. All data were obtained at $200 \%$ elongation.

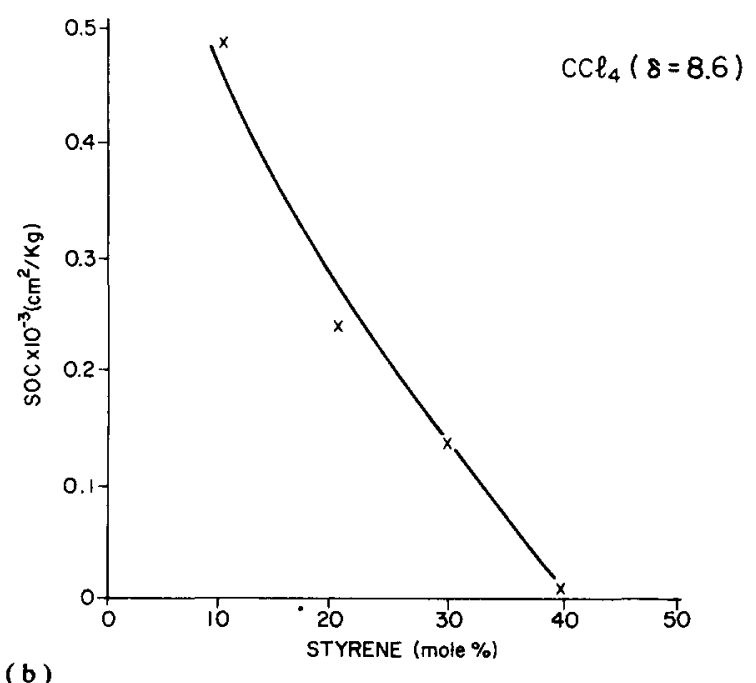

(b) 


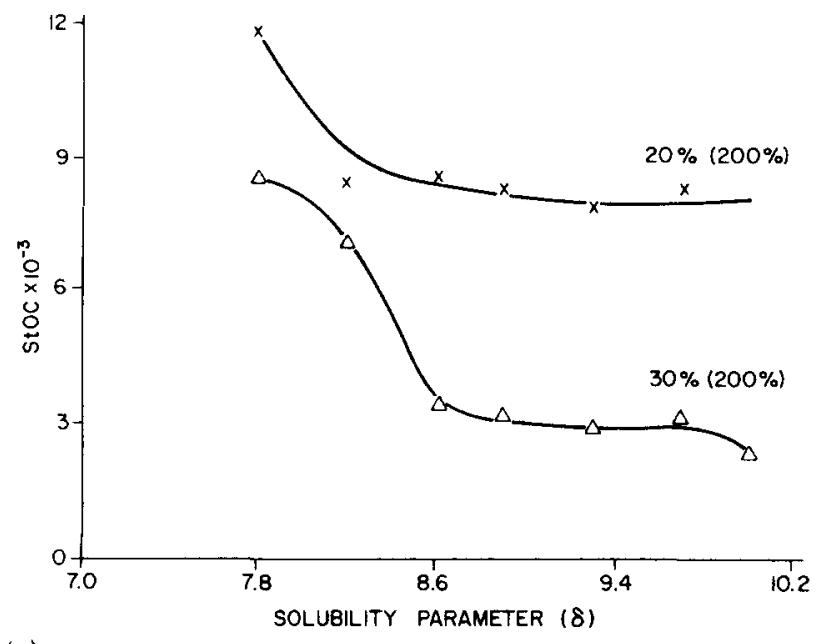

(a)

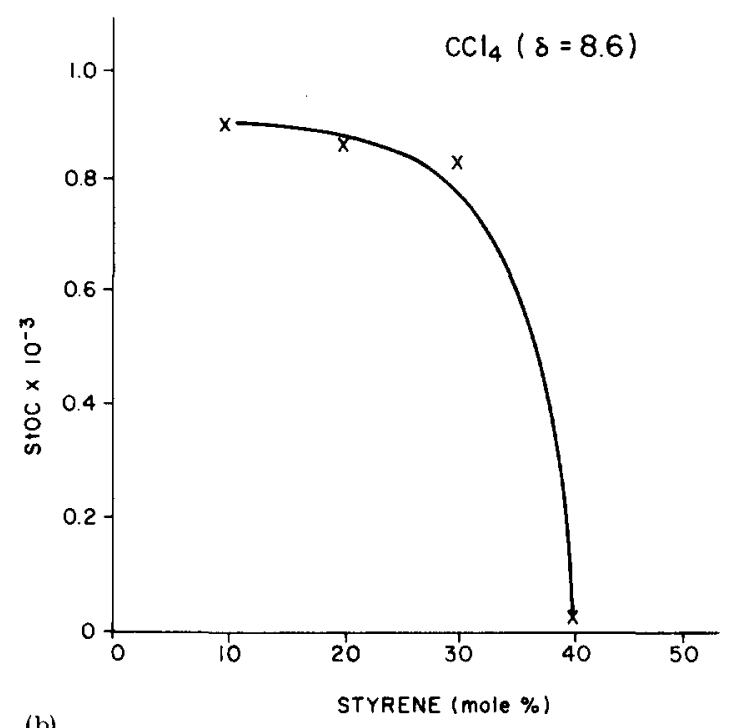

(b)

FIG. 11. ( a ) Plot of the StOC versus mole\% styrene. Films were all cast from carbon tetrachloride, $\delta=8.6$. All data were obtained at 200\% elongation. ( $b$ ) Plot of the StOC versus mole\% styrene. Films were all cast from carbon tetrachloride, $\delta=8.6$. All data were obtained at $200 \%$ elongation.

domains. However, at higher styrene content when the morphology changes to cylindrical or lamellar, the strain should become somewhat more equally distributed so that the parallel model discussed above may be more realistic. Interesiingly, while only four data points exist in Fig. 11(b), it is noted that indeed the behavior of the StOC does not decrease rapidly until above $30 \%$ styrene content for the casting solvent of carbon tetrachloride ( $\delta=8.6$ ). This drop may in fact be reflecting the onset of the morphological change. The second reason for not relying on the above parallel model in this case is that there may also be a third component contributing to Eq. (1). This may be that some of the styrene may be behaving rubberlike if mixed with the butadiene. In this case, its birefringence contribution would be of negative sign due to the negative value of the SOC of rubbery polystyrene. Again this would also cause the StOC to decrease with styrene content. However, unless one knew how the volume fractions of these three "components" change with total styrene content, model calculations would not be particularly useful in describing these systems.

Inspecting Fig. 10( a ), one notes that for a given styrene content, ( 20 or 30 mole\%) at $200 \%$ elongation, the value of the SOC as well as the StOC [Fig. 11( a )] decreases with increasing $\delta$. Indeed, this is also expected if the styrene phase becomes more of a load bearing component for reasons already discussed above. This change can be induced by a change in morphology ( spherical to cylindrical and/or lamellar ) when distinct phase separation is maintained. However, it can in principle be caused by a change in the degree of phase separation such that a fraction of the polystyrene may become rubberlike and thus lower the overall birefringence term in the StOC as well as the SOC parameter. This latter possibility is clearly not the major cause of the change in either SOC or StOC with $\delta$ since the mechanical properties show a stiffening of the material with $\delta$ for most systems. This would not be expected if the polystyrene were being "softened" by mixing with polybutadiene. It is clear, however, that based on the SAXS results, the degree of phase separation is also being influenced by $\delta$. In contrast, it is recalled that the TEM data (for the $30 \%$ styrene material ) do not display a large change in morphology with $\delta$. In summary, therefore, it is clear that the effect of $\delta$ on properties is not necessarily due to a single factor, i.e., (1) a morphological change with no change in the extent of phase separation or ( 2 ) a change in phase separation without a change in morphological texture. Rather, all data tend to promote the speculations that it is likely a combination of these two factors, the magnitude of each which has not been quantitatively determined, but clearly indicated. Furthermore, this study has some practical ramifications in that the nature of solvent medium is crucial in systematically influenceing the final solid-state properties of these block copolymer systems. Thus, any process which is based on solvent use will be prone to the effects dicussed within this work, e.g., solvent-based adhesives. In closing, it is important to add that while the total interpretation of these data have not been given, it is clear that a multitechnique approach has been beneficial. It is likely that in other multiphase or multicomponent polymer systems, this same approach may be necessary in order to best understand the structure property behavior.

'S. Bagrodia and G.L. Wilkes, J. Biomed. Mater. Res. 10, 101 ( 1976 ). 'S.L. Samuels and G.L. Wilkes, Polym. Eng. Sci. 13, 280 ( 1973 ). ${ }^{3}$ G.L. Wilkes, Ph.D. thesis (Univ. of Massachusetts, 1969) ( unpublished ).

${ }^{4}$ G.L. Wilkes and R.S. Stein, J. Polym. Sci. A-2 7, 1525 ( 1969 ).

${ }^{3}$ G.L. Wilkes and S.L. Samuels, in Block and Graft Copolymers, edited by J.J. Burke and V. Weiss ( Syracuse Univ. Press, Syracuse, N.Y., 1973 ), p. 225

${ }^{6}$ E. Fisher and J.F. Henderson, J. Polym. Sci. C 26, 149 ( 1969 ). 'J.F. Beecher, L. Marker, R.D. Bradford, and S. L. Aggarwal, J. Polym. Sci. C 26, 117 ( 1969 ). 
${ }^{8}$ A. Noshay and J.E. McGrath, Block Copolymers ( Academic, New York, 1977).

'See J. Polym. Sci. C 26 ( 1969 ).

${ }^{10}$ A. Beamish, R.A. Goldberg, and D.J. Houston, Polymer 18, 49 ( 1977).

${ }^{11}$ E. Pedemonte, G. Dondero, F. deCandia, and G. Romano, Polymer 17, 72 (1976).

${ }^{12}$ V. Bianchi, E. Pedemonte, and A. Turturro, Polymer 11, 268 ( 1970 ).

${ }^{13}$ T. Soen, T. Inoue, K. Miyoshi, and N. Kawai, J. Polym. Sci. A-2, 10, 1757 ( 1972).
${ }^{14}$ D.J. Meier, in Ref. 5, p. 105.

${ }^{15}$ J.A. Faucher, J. Polym. Sci. A-2 12, 2153 ( 1974 )

${ }^{16}$ T. Miyamoto, K. Kodama, and K. Shibayama, J. Polym. Sci. A-2 8, 2095 (1970).

1'T. Hashimoto, K. Nagatoshi, A. Todo, H. Hasegawa, and H. Kawai, Macromolecules 7, 364 ( 1974 ). 